\title{
Hidretos Parentais, Nomenclatura Aditiva e Substitutiva ... ... Serão Apenas Nomes Novos para Conceitos Velhos?
}

\author{
Bernardo J. Herold ${ }^{1^{*}}$, Adélio A.S.C.M. Machado ${ }^{1}$, João \\ Cardoso $^{2}$, Joaquim Marçalo ${ }^{1}$, José Alberto L. Costa ${ }^{1}$, Maria Clara \\ Magalhães ${ }^{1}$, Maria Helena Garcia ${ }^{1}$, Olivier Pellegrino ${ }^{1}$, Osvaldo \\ Serra ${ }^{3}$, Roberto B. Faria ${ }^{3}$ e Rui Teives Henriques ${ }^{1}$
}

\begin{abstract}
A tribuir nomes a todas as novas substâncias químicas referidas na literatura científica (em média mais de cerca de 12000 por dia) exige um modo de proceder cada vez mais sistemático. Os nomes dados pelos "nomenclaturistas" profissionais parecem muitas vezes estranhos e a sua interpretação é complexa. Uma parte do esforço da União Internacional de Química Pura e Aplicada (IUPAC) de revisão das regras de nomenclatura, de forma a facilitar a dedução das estruturas a partir dos nomes, é criar um conjunto coerente de regras aplicável tanto às substâncias inorgânicas, como às orgânicas e organometálicas. Para apresentar as mesmas de uma forma clara e simples, introduziram-se novos termos, tais como por exemplo hidreto parental, composto parental funcional, descritor, nomenclatura aditiva, nomenclatura substitutiva e nomenclatura permutativa. Mas estas palavras corresponderão apenas a "vinho velho em odres novos" ou a conceitos novos definidos com maior rigor e amplitude que os antigos?
\end{abstract}

Os méritos de cada sistema de nomenclatura revelam-se sobretudo na facilidade com que se deduz a estrutura de cada substância a partir do seu nome. Para facilitar este processo, um passo fundamental é identificar o sistema segundo o qual foi atribuído o nome. Por isso, torna-se cada vez mais importante saber distinguir entre os sistemas substitutivo e aditivo, e conhecer as suas regras e domínios de aplicação. Para a nomenclatura substitutiva, inicialmente desenvolvida para a Química Orgânica, também é indispensável saber o que é um hidreto parental e um composto parental funcional, bem como conhecer a diferença entre nomenclatura substitutiva e permutativa. A nomenclatura aditiva, por sua vez, resultou duma ampliação do método e do domínio de aplicação do sistema de nomenclatura da química de coordenação, criado por Werner. Nos compostos organometálicos e outros em que existem um ou mais átomos centrais, aparecem cada vez mais exemplos em que se usa simultaneamente as nomenclaturas aditiva e substitutiva. Nesses casos, é essencial saber identificar as partes do nome resultantes da aplicação de um e do outro método, o que só é possível através do conhecimento rigoroso destes conceitos.

\section{INTRODUÇÃO}

A Nomenclatura Química, entendida como um conjunto de regras para atribuir nomes a substâncias químicas, é um assunto que, para a esmagadora maioria dos químicos, não é de interesse prioritário. Por isso, muitos químicos limitam-se a conhecer os nomes das substâncias com que mais trabalham dentro do seu domínio de actividade e a dominar um mínimo de

Comissão de Tradução das Recomendações da IUPAC de 2005 da Nomenclatura de Química Inorgânica Portugal

Cabo Verd

Brasil

herold@ist.utl.pt regras necessárias para dar nomes às substâncias novas com que se defrontam. No ensino universitário, a nomenclatura também não é presentemente um tema preferido, seja por estudantes, seja por professores. A divisão do ensino entre Química Inorgânica e Orgânica também não favorece a divulgação duma visão de conjunto de toda a nomenclatura química. O conhecimento pormenorizado da nomenclatura química fica assim demasiado confinado aos "nomenclaturistas" do norte-americano CAS Chemical Abstracts Service, aos membros da Divisão de Nomenclatura Química e Representação
Estrutural (Divisão VIII) e do ICTNS (Interdivisional Committee for Terminology, Nomenclature and Symbols) da IUPAC, aos tradutores dos livros de nomenclatura química da IUPAC $[1,2]$, ao corpo redactorial de publicações periódicas de química, e aos autores e tradutores dos inventários gigantescos de substâncias, como por exemplo os da União Europeia e da Organização Mundial da Saúde. Esta aversão tem como consequência um desconhecimento bastante generalizado dos esforços feitos nas últimas décadas pela IUPAC para tornar a nomenclatura mais racional e mais simples de aprender e aplicar, bem como 
para harmonizar as nomenclaturas inorgânica e orgânica [3]. Quanto a este último aspecto, a própria terminologia usada para os diversos tipos de nomenclatura nos livros de nomenclatura da IUPAC é largamente desconhecida. Quem é o químico inorgânico que sabe o que é o sistema de Hantzsch-Widman de atribuir nomes a heterociclos e conhece a nomenclatura de fusão para policiclos? Quem é o químico orgânico que sabe que a nomenclatura de coordenação é um caso particular da nomenclatura aditiva? Qual é a diferença entre nomenclatura aditiva e substitutiva, e entre esta e a nomenclatura permutativa? O que é um hidreto parental e qual é a utilidade deste conceito e onde se aplica? Qual é a diferença entre as funções de afixos (conceito geral que abrange prefixos, infixos e sufixos) terminados em "o", "a" e "i"? Quando é que são elididas as vogais terminais dos afixos? O que significam os termos "composto parental funcional", "descritor", nomenclatura "aditiva", "substitutiva" e "permutativa"?

Estes saberes pouco atraentes e aparentemente herméticos dirão alguma coisa a pessoas que não sejam "fanáticos da nomenclatura" ("nomenclature freaks")? Sem querer responder a esta pergunta, uma coisa é certa: quando se tenta traduzir e adaptar a uma língua diferente do inglês, que é a única língua oficial da IUPAC, as novas regras de nomenclatura, muitas das pessoas que se sentem repelidas pela tecnicidade excessiva dos textos oficiais de nomenclatura acham que têm o direito de dar opiniões sobre os nomes que resultam da aplicação dessas regras. Não há dúvida que os potenciais utilizadores das regras têm uma palavra a dizer, mas se não se consegue convencê-los a usar os nomes novos baseados numa maior sistematização e racionalização, por entrarem em conflito com os seus hábitos e sensibilidade linguística, todos os esforços para atingir estes objectivos terão sido em vão.

Os especialistas mais experientes no estabelecimento de regras de nomenclatura sabem que o processo de aceitação geral dum método novo de nomenclatura leva muitas vezes o tempo de uma geração, isto é, cerca de 30 anos. No entanto, as regras que são suficientemente sistemáticas para aliviar o esforço de memorização acabam sempre por sair vencedoras desta luta entre a tradição e a racionalidade. Tradicionalmente, as novas gerações de estudantes preferem sempre a compreensão racional à tradição. Se ainda na década de 1960 não se perdoava a um aluno universitário de química desconhecer a estrutura do resorcinol, hoje prefere-se o nome benzeno-1,3-diol do qual resulta imediatamente a fórmula estrutural. Uma pesquisa na internet fornece pelo menos a qualquer pessoa dez sinónimos para este composto. Benzeno-1,3-diol resulta da aplicação de um método extremamente simples que se enquadra na nomenclatura chamada substitutiva: identifica-se primeiro como grupo característico funcional principal (ou simplesmente grupo principal) o grupo hidroxilo, $\mathrm{OH}$, designado pelo sufixo -ol, em segundo lugar identifica-se como "hidreto parental" (neste caso hidrocarboneto "parental") o benzeno, em terceiro lugar junta-seIhe o sufixo -ol e em quarto lugar um prefixo multiplicativo di para o sufixo -ol, dando diol, em quinto lugar os localizadores 1 e 3, obtendo-se assim o nome benzeno-1,3-diol. Obviamente, logo que se tenha ensinado a um jovem estudante este método, este vai oferecer uma sã resistência ao esforço de memorizar o nome resorcinol e igualmente os restantes oito sinónimos. Duvida-se que hoje ainda haja, mesmo entre os alunos mais altamente qualificados, alguém que saiba o que é o resorcinol. Essencial neste método de nomenclatura "substitutiva" é saber que o "grupo principal" substitui um ou mais átomos de hidrogénio num "hidreto parental", que só pode haver um único grupo principal em cada composto, e que, se houver outros grupos funcionais, estes devem ser citados como prefixos, por exemplo, "etanolamina" é um nome inaceitável para 2-aminoetanol. Neste caso, como em 5-aminobenzeno-1,3diol, atende-se a uma lista de prioridades para a atribuição da qualidade de grupo principal a um dado grupo funcional (neste caso o grupo hidroxilo) em comparação com outro.

Embora o exemplo do benzeno-1,3diol seja extremamente simples, não deixa de ser típico para uma situação em que um conjunto de regras de aplicação simples a um número ilimitado de estruturas dispensa a memorização penosa de imensos nomes chamados triviais, como é o caso do resorcinol.

\section{Sistemas de Nomenclatura [4]}

Presentemente distinguem-se, além da nomenclatura meramente composicional, dois sistemas fundamentais de nomenclatura estrutural, embora também hajam outros menos importantes:

\section{Nomenclatura composicional \\ 2. Nomenclatura estrutural \\ 2.1 Nomenclatura substitutiva \\ 2.2 Nomenclatura aditiva \\ 2.3 Outras}

A nomenclatura composicional é usada em nomes que reflectem apenas a composição como, por exemplo,

$\begin{array}{ll}\text { trioxigénio } & \mathrm{O}_{3} \\ \text { óxido de dinitrogénio } & \mathrm{N}_{2} \mathrm{O} \\ \text { cloreto de hidrogénio } & \mathrm{HCl} \\ \text { sulfato de ferro(3+) } & \mathrm{Fe}_{2}\left(\mathrm{SO}_{4}\right)_{3} \\ \text { tricloreto de fósforo } & \mathrm{PCl}_{3}\end{array}$

Nos casos mais simples, para cada composição há apenas uma estrutura possível, pelo que se dispensa um nome que explicite a forma como os átomos estão ligados uns aos outros. A nomenclatura composicional actual resultou de aperfeiçoamentos introduzidos gradualmente ao longo de mais de dois séculos no sistema concebido por G. de Morveau, A.L. Lavoisier, C.-L. Berthollet e A.F. de Fourcroy em 1787 [5], texto que foi traduzido para português por Vicente Coelho de Seabra Telles em 1801 [6].

No último quartel do século XIX, com a vitória do "atomismo" sobre o "equivalentismo" e a crescente aceitação das doutrinas de valência de Kékulé, Couper e Butlerov, surgiu pela primeira vez a necessidade de se criar um sistema para atribuir nomes a compostos que traduzissem a sua estrutura, portanto uma nomenclatura que se pode designar de "estrutural". Como as primeiras estruturas que se conseguiram determinar foram as de compostos orgânicos, criou-se em 1892, num congresso em Genebra, regras de nomenclatura da Química 
Orgânica que foram aperfeiçoadas e expandidas ao longo de todo o século $\mathrm{XX}$. Só muito mais tarde é que se cunharam os termos "nomenclatura substitutiva" e outros para diferenciar os vários tipos de nomenclatura estrutural que foram surgindo. A nomenclatura estrutural da Química Inorgânica só se desenvolveu a partir do momento em que Werner lançou os fundamentos de processos de determinação de estrutura dos compostos de coordenação.

\section{Nomenclatura Substitutiva}

Para quem esteja menos familiarizado com a nomenclatura química podem, no entanto, ainda parecer estranhas algumas das designações recentes como "nomenclatura substitutiva", "hidreto parental", "grupo principal", "prefixo multiplicativo", "localizador", "nomenclatura permutativa", etc..

Para as compreender melhor, é preciso explicar que aquilo que hoje se chama nomenclatura substitutiva resultou do desenvolvimento das já referidas regras de nomenclatura de compostos orgânicos fixadas em Genebra em 1892. Quando se começou a aplicar por analogia o mesmo método a compostos de outros elementos, como por exemplo aos do silício e do fósforo, isto é, quando se passou dos alcanos para compostos de outros elementos dos grupos 13 a 17, como sejam, por exemplo os silanos, estananos, fosfanos, etc., os nomes passaram a ser derivados não só a partir de hidrocarbonetos, como sejam os alcanos, mas também dos silanos, etc..

Ao formular as regras de uma forma generalizada, passou-se por isso a substituir a designação inglesa parent hydrocarbon (hidrocarboneto parental) pela mais geral de parent hydride (hidreto parental). A origem do termo hidrocarboneto parental é a designação alemã usada por Richter e Beilstein no início do século XX, Stammkohlenwasserstoff. O sistema de Richter de classificação dos compostos orgânicos esteve na base da organização do grande tratado de Química Orgânica de Beilstein e deixou uma marca indelével no desenvolvimento da nomenclatura internacional da Química
Orgânica. Em português, os tradutores do Guia da Nomenclatura IUPAC da Química Orgânica [1] optaram por traduzir parent ou o prefixo Stamm pelo adjectivo parental, um termo cujo uso com o significado "relativo a pai e mãe" está documentado em português desde $1817^{1}$.

Hoje, o uso deste método de nomenclatura, que era inicialmente exclusivo da Química Orgânica, é recomendado pela IUPAC para os compostos dos elementos dos grupos 13 a 17, cujas estruturas podem ser derivadas por substituição dum átomo de hidrogénio num composto desse elemento com hidrogénio. Daí ter-se deixado de considerar esse método só de "nomenclatura da química orgânica" e se ter optado pelo termo mais geral "nomenclatura substitutiva", bem como passar a usar-se "hidreto parental" em lugar de "hidrocarboneto".

Uma das grandes vantagens do método substitutivo resulta de dispensar localizadores para os átomos de hidrogénio que não foram substituídos. Em compostos com um número elevado de átomos de hidrogénio, a dispensa desses localizadores resulta em nomes muito mais simples.

O mesmo método aplica-se assim a casos do género dos exemplificados a seguir, em que em vez de carbono se tem outro elemento dos grupos 13 a 17.

As estruturas são deduzidas dos nomes por um processo perfeitamente análogo ao dos hidrocarbonetos:

2-metilpentassilano

$\mathrm{SiH}_{3}-\mathrm{SiH}\left(\mathrm{CH}_{3}\right)-\left(\mathrm{SiH}_{2}\right)_{2}-\mathrm{SiH}_{3}$

por analogia com 2-metilpentano

$\mathrm{CH}_{3}-\mathrm{CH}\left(\mathrm{CH}_{3}\right)-\left(\mathrm{CH}_{2}\right)_{2}-\mathrm{CH}_{3}$

3-clorononafosfano

$\mathrm{PH}_{2}-\mathrm{PH}-\mathrm{PCl}-(\mathrm{PH})_{5}-\mathrm{PH}_{2}$

por analogia com 3-clorononano

$\mathrm{CH}_{3}-\mathrm{CH}_{2}-\mathrm{CHCl}-\left(\mathrm{CH}_{2}\right)_{5}-\mathrm{CH}_{3}$

Quando há um ou mais substituintes que podem desempenhar o papel de grupo característico principal, o de mais elevada prioridade dá origem a um sufixo, como "ol" em: 3-metilciclopentassilanol

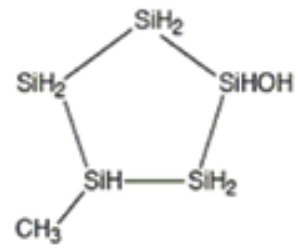

por analogia com:

3-metilciclopentanol

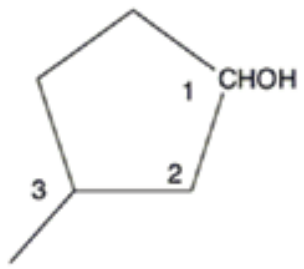

É essencial ter em atenção que tanto a prefixação como a sufixação se fazem por substituição de um ou mais átomos de hidrogénio de um hidreto parental.

A designação "nomenclatura substitutiva" marca claramente a distinção de outros métodos de nomenclatura química, nomeadamente da "nomenclatura aditiva".

\section{Nomenclatura Permutativa}

Um sistema muito utilizado em conjugação com a nomenclatura substitutiva (em inglês substitutive nomenclature) é a chamada nomenclatura permutativa (em inglês replacement nomenclature), em que se deriva o nome dum hidreto parental de outro, permutando por exemplo na cadeia de um hidrocarboneto um grupo $\mathrm{CH}_{2}$ por um grupo $\mathrm{SiH}_{2}$. Assim se deriva a partir do ciclopentano o silaciclopentano:

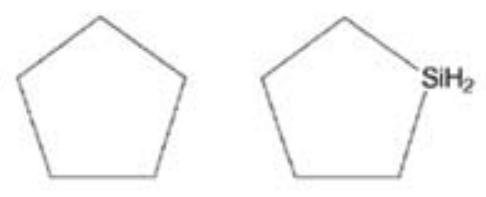

Esta não deve ser confundida com a nomenclatura substitutiva em que se substituem átomos de hidrogénio. No presente caso, os prefixos de permuta terminam todos em 'a', por exemplo 'oxa', 'sulfa', 'aza', 'fosfa', etc.. 


\section{Nomenclatura Aditiva}

A nomenclatura chamada aditiva usase em Química Orgânica quando se deriva um nome dum composto a partir do correspondente composto insaturado, por exemplo, a partir de naftaleno obtém-se o nome 1,2,3,4tetra-hidronaftaleno para o composto formado por adição de quatro átomos de hidrogénio.
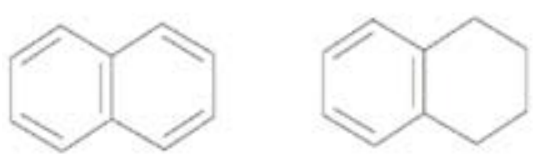

Verificou-se mais tarde que o conceito de "nomenclatura aditiva" afinal também podia ser aplicado à nomenclatura proposta por Werner para compostos de coordenação no âmbito da Química Inorgânica. O conceito alargado de nomenclatura aditiva inclui assim a nomenclatura de Werner que tinha como ponto de partida um "átomo central" ao qual se ligam átomos ou agrupamentos de átomos designados por "ligandos". Essa operação tem o carácter aditivo, contrariamente às operações substitutivas (de átomos de hidrogénio) mais comuns na Química Orgânica. Com o passar do tempo, o método característico da nomenclatura de coordenação foi sendo aplicado a outras entidades, como sejam ácidos inorgânicos e compostos organometálicos. Ao atingir esse ponto, já não fazia sentido continuar a usar o termo restritivo "nomenclatura de coordenação" e foi então que se propôs o termo novo para o sentido alargado de "nomenclatura aditiva". A aplicação da nomenclatura aditiva aos ácidos inorgânicos e às suas bases conjugadas tem a vantagem de conduzir a nomes mais sistemáticos que certos nomes tradicionais que, no passado, tinham dado origem à proliferação de sinónimos e a muitas ambiguidades. Na nomenclatura de compostos organometálicos tornouse então absolutamente indispensável. Nesta última deu-se porém, como era de esperar, o choque das culturas de nomenclatura orgânica e inorgânica. Conforme a cultura originária dos investigadores, os nomes propostos muitas vezes diferiam. Acresce que, em compostos organometálicos relativamente complicados, é muitas vezes inevitável usar simultaneamente as nomenclaturas aditiva e substitutiva. Num nome de base aditiva podem assim aparecer partes respeitantes a certos ligandos que resultam da aplicação da nomenclatura substitutiva. Para decifrar um nome complicado desses, é preciso primeiro que tudo identificar as várias partes. Antes de mais nada, tem de se identificar o átomo central e a seguir os ligandos. Esta tarefa é relativamente simples em compostos de coordenação não organometálicos, como ilustra o seguinte exemplo:

pentacloretonitreto-osmato(2-) de potássio

$\mathrm{K}_{2}\left[\mathrm{OsCl}_{5} \mathrm{~N}\right]$

Exemplo dum nome aditivo dum ácido:

hidreto-hidroxido-oxidoenxofre

(em lugar de ácido sulfínico)

$\mathrm{SHO}(\mathrm{OH})$

Continua a ser simples dar nomes a compostos organometálicos em que os ligandos têm nomes que são derivados de nomes de compostos orgânicos não substituídos:

\section{Tricloreto(metilo)titânio}

Tricloreto(metaneto)titânio

O segundo nome é obtido se se atribuir a carga formal 4+ ao átomo de titânio.

Quando há nomes de ligandos que são derivados dos de compostos orgânicos substituídos, a tarefa tornase mais complicada, porque um determinado grupo, por exemplo metilo, tanto pode aparecer como ligando, isto é, ligado directamente ao átomo central, como também como substituinte dentro da estrutura de um dos ligandos, quando o nome do ligando é derivado do nome dum hidreto parental pelo método substitutivo. No primeiro caso, o grupo metilo não está a substituir nenhum átomo de hidrogénio, mas no segundo isto já não é o caso. Enquanto na nomenclatura substitutiva se elide a vogal terminal do nome do grupo substituinte, por exemplo o 'o' em fenilo, dando portanto fenilfosfano, na nomenclatura aditiva adicionam-se os nomes dos ligandos sem nenhuma alteração, não elidindo a mesma vogal terminal. Isto simplifica o processo de dedução da estrutura a partir do nome, uma vez que se conclui automaticamente que os grupos com prefixos terminados em 'ilo' estão ligados directamente ao átomo central, enquanto que os terminados em 'il' substituem um átomo de hidrogénio na estrutura dum ligando cujo nome se deriva dum hidreto parental.

(butano-1,4-di-ilo)bis(trifenilfosfano) platina2:

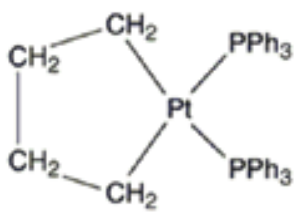

(1,2-difenil-3-oxopropano-1,3-di-ilo) bis(trifenilfosfano)platina:

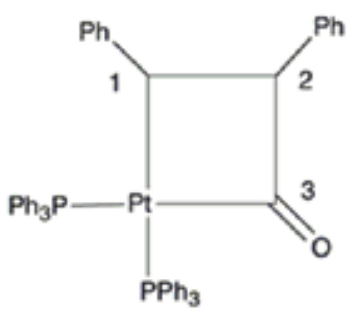

(2,4-dimetilpenta-1,3-dieno-1,5-di-ilo) tris(trietilfosfano)irídio(1+):

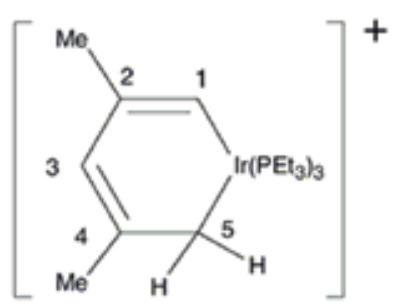

tetracarbonilo[2-(2-fenildiazen-1-ilo$\mathrm{KN}^{2}$ )fenilo-kC ${ }^{1}$ ]manganês:

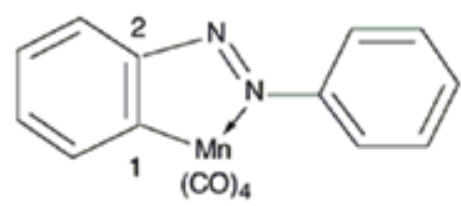

cloreto-hidreto(2-metil-3-oxo-kO-but1-en-1-ilo)bis(tri-isopropilfosfano) ródio:

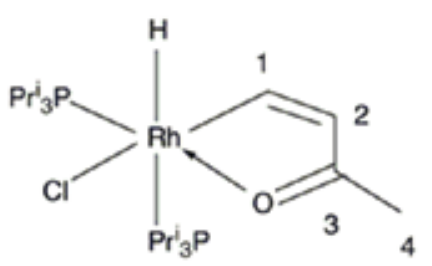


( $\mu$-etano-1,1-di-ilo)bis(pentacarbonilorénio):

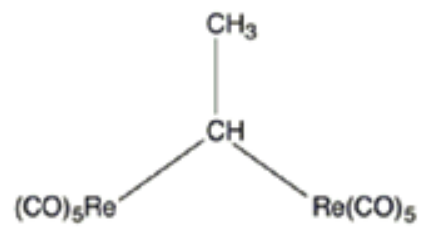

\section{Observações Finais}

Espera-se que este artigo desfaça algumas dúvidas sobre os desenvolvimentos recentes da nomenclatura química da IUPAC. Só com o esclarecimento dessas dúvidas é possível conseguir que os químicos se convençam a utilizá-la, rompendo com hábitos enraizados. Além disso, para traduzir bem em português as regras publicadas em inglês pela IUPAC, também é indispensável conhecer os propósitos que levaram os seus autores, em cada caso, a formulá-las de uma certa maneira quando havia outras opções aparentemente possíveis. Ao longo do caminho que se percorre quando se traduz e adapta a nomenclatura química à língua portuguesa, aparecem novamente bifurcações. A escolha da opção preferível num caso particular é mais fácil para quem tenha uma visão do conjunto completo dos sistemas de nomenclatura. Foram essas as razões que levaram os autores a submeter este ensaio à consideração da comunidade química de língua portuguesa.

\section{Notas}

${ }^{1}$ Actualmente também se usa com o significado "relativo a parentes".

${ }^{2}$ Nas fórmulas moleculares que representam estruturas constituídas por um átomo central e vários ligandos, este conjunto é sempre colocado entre parênteses rectos, quer tenha uma carga eléctrica, quer não. Nas fórmulas de estrutura, por uma questão de simplificação gráfica, poderão omitir-se os parênteses nos compostos neutros.

\section{REFERÊNCIAS}

[1] Guia IUPAC para a Nomenclatura de Compostos Orgânicos, Tradução Portuguesa nas Variantes Europeia e Brasileira, (R. Panico, W. H. Powell, J.-C. Richer, A Guide to IUPAC Nomenclature of Organic Compounds Recommendations 1993, Blackwell Scientific Publications, Oxford 1993) Tradução de A. C. Fernandes, B. Herold, H. Maia, A. P. Rauter, J. A. R. Rodrigues, Lidel Edições Técnicas, Lda. Lisboa 2002.

[2] Nomenclatura de Hidratos de Carbono, Tradução Portuguesa nas Variantes
Europeia e Brasileira de Nomenclature of Carbohydrates (IUPAC and IUBMB Recommendations 1996), original Inglês preparado para publicação por Alan D. McNaught, Tradução Portuguesa para a Sociedade Portuguesa de Química de A. P. Rauter, B. J. Herold, A. Moreira da Silva, Â. Relva, J. A. Figueiredo, J. A. Rosário Rodrigues, L. Bento, M. Caldeira, M. A. Coimbra, M. I. Ismael, Lidel - Edições Técnicas, Lda. Lisboa 2010.

[3] N. G. Connelly, T. Damhus, R. M. Hartshorn, A. T. Hutton, Nomenclature of Inorganic Chemistry, IUPAC Recommendations 2005, RSC Publishing, Cambridge 2005.

[4] G. J. Leigh, H. A. Favre, W. V. Metanomski, Principles of Chemical Nomenclature, Blackwell Science, Oxford 1998. Nova edição: RSC, no prelo.

[5] G. de Morveau, A. L. Lavoisier, C.-L. Berthollet et A. F. de Fourcroy, Nomenclature Chimique, Chez Cuchet, Sous le Privilège de l'Académie des Sciences, 1787 (251 pg.)

[6] V. Coelho de Seabra S. Telles, Nomenclatura Chimica Portugueza, Franceza e Latina, a que se ajunta o Systema de Characteres Chimicos adaptados a esta Nomenclatura por Hassenfratz e Adet, Typographia Chalcographica, Typoplastica e Litteraria do Arco do Cego, Lisboa 1801. (125 pg.)

\section{Actualidades Científicas}

\section{Activação de Metano por Reagente Organometálico}

Cientistas do Estados Unidos demonstraram pela primeira vez que o metano pode ser activado por um complexo com ligações múltiplas metal-carbono. O trabalho pode abrir caminho para a conversão eficiente de metano em hidrocarbonetos úteis, incluindo etileno, uma das matérias primas fundamentais da indústria química. O metano é o principal componente do gás natural e um dos maiores contribuidores para o aquecimento global. É por isso desejável encontrar formas mais limpas e baratas de usá-lo como matéria prima na produção de compostos químicos mais úteis. No entanto, tais reacções constituem desafios significativos, devido à baixa reactividade do metano e às fortes ligações $\mathrm{C}-\mathrm{H}$ tetraédricas, que não são facilmente acessíveis para ataque químico e activação.

Embora existam reagentes conhecidos que activam o metano para conversão química, são poucos em número, produzem baixos rendimentos e operam sob condições extremas que requerem elevadas temperaturas ou a utilização de materiais caros. No entanto, estudos teóricos e cinéticos recentes sugerem que a activação do metano é possível com ligações múltiplas metal-carbono actuando como intermediários reactivos. Daniel Mindiola da Universidade de Indiana, em Bloomington, e colegas, mostraram experimentalmente isto pela primeira vez, demonstrando uma via possível que utiliza um complexo contendo uma ligação tripla titânio-carbono para activar a ligação $\mathrm{C}-\mathrm{H}$ do metano sob condições amenas $\left(25-30^{\circ} \mathrm{C}\right)$.

Mindiola sugere que esta descoberta pode permitir a utilização futura de metano como matéria prima para a formação de ligações C-C, importante para a produção de produtos químicos, incluindo compostos farmacêuticos e plásticos.

(adaptado de http://www.rsc.org/chemistryworld/News/2011/June/02061103.asp)

Helder Gomes (bquimica@ipb.pt) www.spq.pt 
$3^{\text {rd }}$ PYCheM

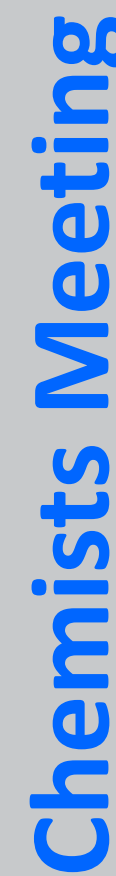

$\frac{b}{3}$

(1)

幽
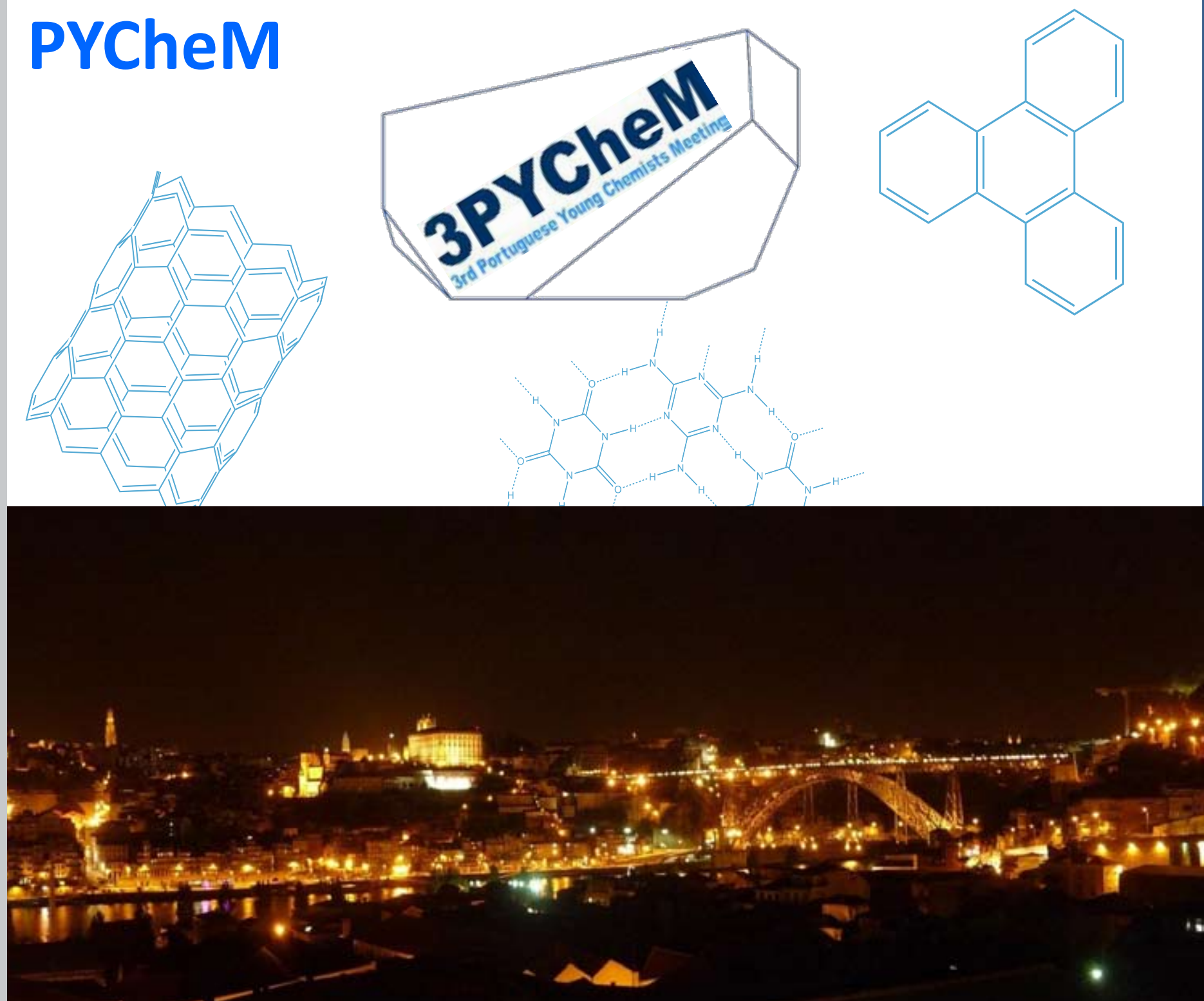

물

9 - 11 Maio 2012
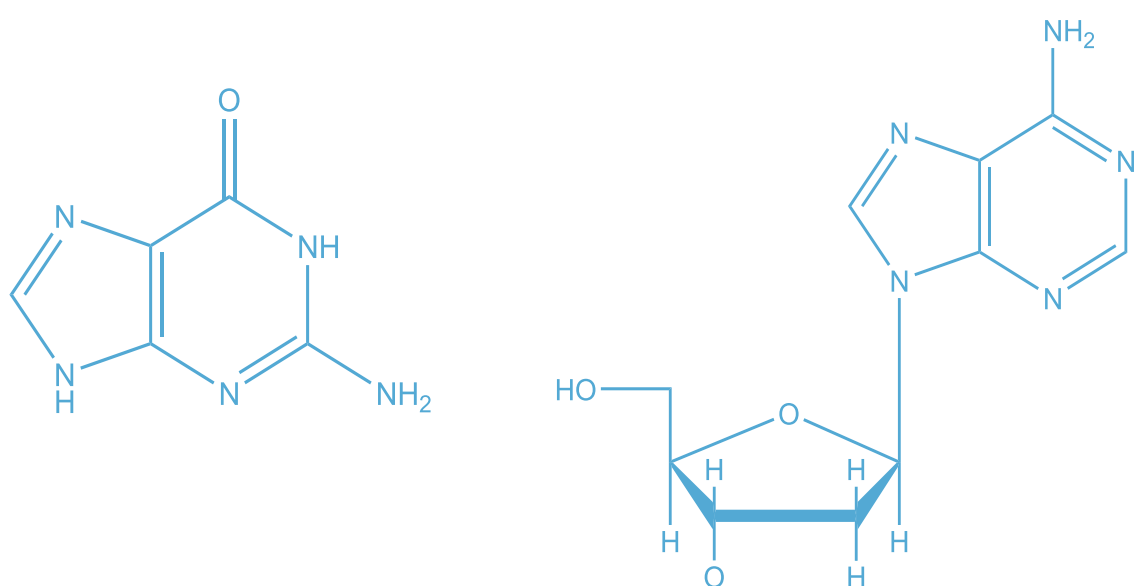

\section{Departamento de Química e Bioquímica}

Faculdade de Ciências da Universidade do Porto

Contacto: 3pychem@spq.pt

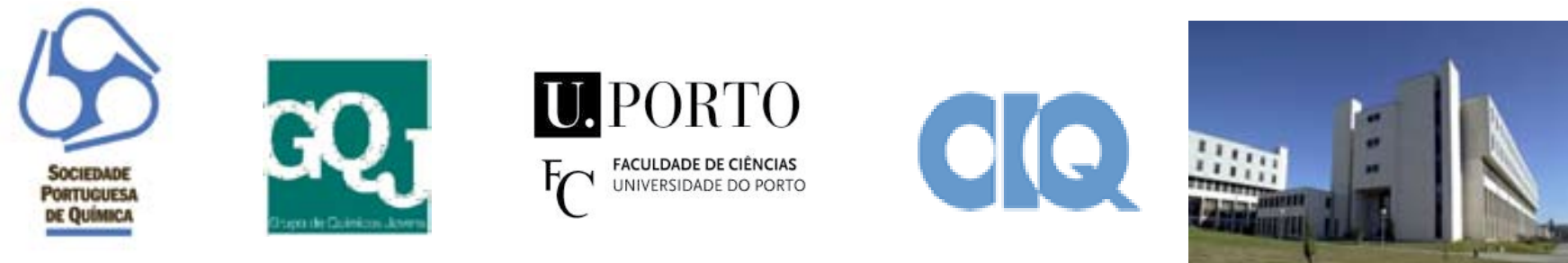

